\title{
Custo do tratamento de idosos com pneumonia em terapia intensiva
}

\author{
João Batista Feitosa*, Hercília Najara Ferreira de Souza**, Talline Aredes Hang Costa**, \\ Alessandra Figueiredo de Souza***, Maria Thereza Fonseca Martins ${ }^{* * *}$, Ana Cristina Viana Campos*****
}

*Enfermeiro, Especialista em Unidade de Terapia Intensiva pelo Instituto de Educação Continuada Pontifícia Universidade Católica de Minas Gerais, **Enfermeira, Pós graduanda em Terapia Intensiva Adulto pelo Centro Universitário UMA, ${ }^{* * *}$ Cirurgiā-dentista, ${ }^{* * * *}$ Cirurgiä-dentista, Doutoranda em Saúde Coletiva pela Faculdade de Odontologia da Universidade Federal de Minas Gerais, Professor do Instituto de Educação Continuada Pontifícia Universidade Católica de Minas Gerais

\section{Resumo}

Sabendo-se das limitaçóes de financiamento público de saúde e da alta demanda por tratamento intensivo, o presente estudo teve como objetivo descrever os gastos diários de um hospital da rede pública de saúde com o tratamento intensivo de idosos com diagnóstico de pneumonia comunitária. Este é um estudo de casos com análise documental em Unidade de Terapia Intensiva de um hospital da rede pública de alta complexidade. A amostra coletada para estudo é composta de prontuários de três pacientes com idade superior a 60 anos internados de $1^{\circ}$ de setembro de 2009 até 31 de dezembro de 2009. Para mensurar o valor dos custos com o tratamento dos pacientes internados com pneumonia foram listados todos os materiais, medicamentos, exames, diárias e demais despesas que cada paciente teve no seu período de internação. Foram excluídos os gastos referentes aos recursos humanos, pelo constante rodízio de profissionais que acompanharam esses pacientes. A média de gastos diários para os Pacientes 1, 2 e 3 foi de $R \$ 480,78( \pm 324,99), R \$ 134,89( \pm 16,07)$ e $R \$ 425,4( \pm 347,45)$, respectivamente. O paciente 3 apresentou uma diferença de $\mathrm{R} \$ 1.073,79$ entre os gastos diários mínimo e máximo. O paciente que teve maior período de internação apresentou maior média de gasto diário e este gasto esteve associado ao agravamento do quadro clínico.

Palavras-chave: unidades de terapia intensiva, gastos em saúde, hospitalização, pneumonia, idoso.

\section{Abstract}

\section{Cost of treatment of elderly patients with pneumonia in intensive care}

Knowing the limitations of public health and the high demand for intensive care, this study aimed to describe the daily expenses of a public hospital with elderly in intensive care with a diagnosis of community-acquired pneumonia. This case study is to document analysis in the intensive care unit of a public hospital of high complexity. A sample collected for the study consists of three records of patients older than 60 years admitted to September 1, 2009 through December 31, 2009. To measure the value of the treatment costs of patients hospitalized with pneumonia were listed all the materials, drugs, tests, 
and other daily expenses in the period of hospitalization. We excluded costs related to human resources, due to the constant rotation of professionals who have followed these patients. The average daily spending for Patients 1, 2 and 3 was $\mathrm{R} \$ 480.78$ ( \pm 324.99), $\mathrm{R} \$ 134.89( \pm 16.07)$ and $\mathrm{R} \$ 425.4$ ( \pm 347.45), respectively. Patient 3 showed a difference of $\mathrm{R} \$ 1,073.79$ between the minimum and maximum daily expenses. The patient who had longer hospitalization had a higher average daily expenditure and this expenditure was associated with worsening of clinical symptoms.

Key-words: intensive care units, health expenditures, hospitalization, pneumonia, aged, cross infection.

\section{Resumen}

\section{El costo del tratamiento de pacientes ancianos con neumonía en cuidados intensivos}

Conociendo las limitaciones de la salud pública y la alta demanda de cuidados intensivos, este estudio tuvo como objetivo describir los gastos diarios de un hospital público con la salud de ancianos en cuidados intensivos con el diagnóstico de neumonía comunitaria. Este es un estudio de casos con análisis documental en unidad de cuidados intensivos de un hospital público de alta complejidad. La muestra colectada para el estudio está compuesta de prontuarios de tres pacientes mayores de 60 ańos ingresados desde el 01 de septiembre 2009 al 31 de diciembre de 2009. Para determinar los costos de tratamiento de los pacientes hospitalizados con neumonía se listaron todos los materiales, medicamentos, exámenes y otros gastos diarios que cada paciente tuvo en el periodo de hospitalización. Se excluyeron los costos relacionados con los recursos humanos, por la rotación constante de profesionales que atendieron estos pacientes. El gasto promedio diario de los pacientes 1, 2 y 3 fue de $\mathrm{R} \$ 480,78( \pm 324,99), \mathrm{R} \$ 134,89( \pm 16,07)$ y $\mathrm{R} \$ 425,4( \pm 347,45)$, respectivamente. El paciente 3 mostró una diferencia de $\mathrm{R} \$ 1,073.79$ entre los gastos mínimos y máximos diarios. El paciente que tuvo mayor tiempo de hospitalización tuvo un mayor gasto medio diario y este gasto se asoció con el empeoramiento de los síntomas clínicos.

Palabras-clave: unidades de terapia intensiva, gastos en salud, hospitalización, neumonía, anciano.

\section{Introdução}

A maior longevidade da população, a crescente utilização de fármacos imunossupressores e o desenvolvimento de novos procedimentos médicos intervencionistas, modificaram a interação hospedeiro-agente infeccioso, favorecendo a emergência de novos microrganismos e o desenvolvimento de patógenos multirresistentes. A ausência de um padrão definido para o diagnóstico que possa ser usado de rotina, o uso indiscriminado de antimicrobianos, e o tratamento empírico inadequado, são fatores que também alicerçam este permanente desafio [1].

Nesse processo, percebe-se uma transição demográfica com consequente alteração do perfil de morbimortalidade marcado pelo predomínio das doenças crônico-degenerativas e suas complicações [2] e as causadas por fatores externos [3]. Para o sistema de saúde isso pode significar décadas de utilização dos serviços (medicamentos, consultas médicas e internações hospitalares de longa duração) acarretando cada vez maiores gastos $[2,4]$.

Enquanto as infecçóes agudas possuem desfechos que normalmente acontecem em um curto período de tempo, as doenças crônicas pressupóem intervençóes que determinem consequências ob- servadas no longo prazo [5]. Entretanto, no Brasil, pode ser evidenciada uma sobreposição das duas categorias de enfermidades, mostrando o surgimento de uma nova demanda nas instituiçóes de saúde em busca de prevenção e tratamento [6].

A cobrança de atendimentos prestados aos pacientes provenientes do Sistema Único de Saúde (SUS) brasileiro é realizada por meio de conta pacote, assim, a cobrança é feita por meio de um valor único que contempla todos os custos e a margem de lucratividade para realização de cada procedimento não estando incluídos gastos excedentes gerados em decorrência do processo de hospitalização [7].

Em serviços de saúde, a gestão de custos tem um papel fundamental para que o processo administrativo seja eficaz e para que o planejamento, controle e acompanhamento dos gastos em saúde se tornem mais efetivos $[8,9]$.

Um estudo realizado em hospital escola em Sáo Paulo revelou que $87,7 \%$ do custo total glosado pelos de planos de saúde pode ser revertido a partir da análise das anotaçóes de enfermagem [10]. O enfermeiro como gestor dos processos em saúde precisa contribuir para a avaliação econômica da saúde e buscar conhecer as características clínicas das doenças e das intervençóes. Tudo isso para que se 
estruture um modelo que capture todos os desfechos importantes na história natural da doença $[8,11]$.

Sabendo-se a necessidade da gestão dos gastos nos serviços de saúde, das limitaçôes de financiamento do SUS e da crescente demanda por cuidados intensivos, torna-se importante investigar o custo do tratamento de pacientes internados em unidades de terapia intensiva. Nesse contexto, o presente estudo teve como objetivo descrever os gastos diários de um hospital da rede pública de saúde com o tratamento intensivo de idosos com diagnóstico médico de pneumonia comunitária.

\section{Material e métodos}

Trata-se de um estudo de casos documental para analisar o custo de pacientes idosos com diagnóstico de pneumonia comunitária internados em Unidade de Terapia Intensiva (UTI) de um hospital da rede pública de alta complexidade em Belo Horizonte/MG.

O hospital possui 44 leitos ativos de clínica médica, 10 para cuidados intensivos e 10 para tratamento intensivo. O Centro de Tratamento Intensivo foi implantado recentemente, com a finalidade de ampliar o suporte de atendimento, náo só aos pacientes internados na enfermaria, caso venham a agravar o quadro clínico como também a outros usuários da rede assistencial de saúde. $\mathrm{Na}$ assistência direta ao paciente internado, o hospital conta com o apoio de uma equipe multidisciplinar - médicos, enfermeiros, técnicos de enfermagem e de laboratório, além de fisioterapeutas que trabalham no atendimento a pacientes com comprometimentos respiratórios ou que necessitam de reabilitação motora.

Integram este estudo pacientes internados na unidade de terapia intensiva com diagnóstico médico de pneumonia comunitária. Os critérios de inclusão foram: 1) pacientes com idade superior a 60 anos; 2) data de internação entre $1^{\circ}$ de setembro de 2009 e 31 de dezembro de 2009; 3) diagnóstico definido de pneumonia comunitária anterior à internação em UTI. Ao final, a populaçáo deste estudo totalizou 3 pacientes.

Para coleta de dados, utilizaram-se informaçóes provenientes das evoluçóes multidisciplinares integrantes do prontuário do paciente. Para mensurar o valor dos custos com o tratamento foram listados todos os materiais, medicamentos, exames, diárias e demais despesas durante a internação. Foram excluídos os gastos referentes aos recursos huma- nos, pelo quantitativo elevado de profissionais em regime de plantáo e constante rodízio profissional no acompanhamento dos pacientes.

Inicialmente, foi realizada a análise descritiva dos gastos diários (custos em Reais) para cada paciente. O gráfico de tendência foi utilizado para evidenciar a comparação entre a média diária de gastos e o gasto diário de cada paciente. Para fins de preservaçáo da identidade dos pacientes, utilizaram-se os codinomes Paciente 1; Paciente 2; Paciente 3, respeitando os princípios éticos.

O banco de dados foi construído no Programa Excel for Windows versão 2007, onde foi realizada análise descritiva e construção dos gráficos.

O projeto foi aprovado pelo Comitê de Ética em Pesquisa da Pontifícia Universidade Católica de Minas Gerais (CAAE - 1013.0.000.213-10), respeitando as Diretrizes da Resoluçáo 196/96 que rege os preceitos da pesquisa em seres humanos no Brasil [12].

\section{Resultados e discussão}

Os pacientes selecionados para este estudo tem idade superior a 60 anos. Esta escolha delimita uma parcela especial da população com maior susceptibilidade às doenças crônicas e elevada procura por serviços de saúde de alta complexidade $[4,13]$. O desenvolvimento tecnológico de novas estratégias diagnósticas e terapêuticas, o envelhecimento da populaçáo e o aumento da expectativa de vida sáo os principais responsáveis pelo aumento dos gastos em saúde [14].

O presente estudo incluiu pacientes hospitalizados para tratamento de pneumonia adquirida na comunidade, com o objetivo de descrever os gastos no período de internação, bem como acompanhar o desfecho de cada paciente, uma vez que as infecçóes pulmonares, sobretudo a pneumonia, são importantes causas de morbimortalidade. No Brasil, constata-se pelo registro necrológico que as doenças respiratórias são responsáveis por aproximadamente $12 \%$ das mortes notificadas no país, sendo a pneumonia responsável por quase metade desses óbitos [15].

A média de gastos diários para os Paciente 1, Paciente 2 e Paciente 3 foi de $\mathrm{R} \$ 480,78( \pm 324,99)$, $\mathrm{R} \$ 134,89$ ( $\pm 16,07)$ e $\mathrm{R} \$ 425,4$ ( $\pm 347,45)$, respectivamente. $\mathrm{O}$ paciente 3 apresentou uma diferença de $\mathrm{R} \$ 1.073,79$ entre os gastos diários, mínimo e máximo (Tabela I). 
Tabela I - Medidas de resumo para gastos diários de cada paciente durante o tempo de permanência na Unidade Intensiva.

\begin{tabular}{lccc}
\hline $\begin{array}{c}\text { Medidas } \\
\text { de resumo* }\end{array}$ & Paciente 1 & Paciente 2 & Paciente 3 \\
\hline Mínimo & 246,07 & 119,47 & 110,12 \\
Média & 480,78 & 134,89 & 425,4 \\
Desvio-padrão & 324,99 & 16,07 & 347,45 \\
Máximo & 953,14 & 171,2 & $1.183,91$ \\
\hline Total & $3.365,48$ & $1.348,89$ & $31.054,53$ \\
\hline
\end{tabular}

* Valores apresentados em Reais (R\$).

A duração média de internação dos pacientes deste estudo foi maior do que a média geral hospitalar. Um estudo de caso semelhante a este encontrou $66,7 \%$ dos pacientes internados com pneumonia comunitária e tinham idade superior a 60 anos, com presença frequente de doença crônica subjacente. Possivelmente, a alta permanência hospitalar possa também ser explicada por esses dois fatores [16].

Cerca de 1/5 das internaçóes hospitalares ocorrem por causa de doenças respiratórias, sendo que os pacientes com doença pulmonar obstrutiva crônica representam a maior parcela dos pacientes, seguidos pelos acometidos por pneumonias e asma brônquica [8].

A análise de custos de cada um baseia-se nos gastos diários que foram quantificados através das prescriçóes e evoluçóes da equipe multidisciplinar. Incluem-se os recursos com materiais, medicaçóes, diárias de monitores, respiradores e insumos de procedimentos invasivos.

Esse tema merece destaque no cenário em que o controle de gastos públicos é realizado de maneira indireta, pela limitação do número de internaçóes e pela definição dos custos por tipo de procedimento pelo sistema público de saúde [17]. O Gráfico 1 refere-se ao Paciente 1 do sexo masculino com 70 anos de idade que permaneceu internado durante sete dias evoluindo a óbito. Observa-se que nos três primeiros dias de internaçáo na UTI os gastos ficaram inferiores à média e que após o quarto dia houve uma ascensão nos gastos, coincidindo com a necessidade de duas sessóes de hemodiálise no valor de $\mathrm{R} \$ 680,00$ cada uma, devido à gravidade do quadro clínico do paciente.

O segundo paciente (Gráfico 2) era do sexo feminino com 71 anos de idade, apresentando permanência de 10 dias. A evolução do seu prognóstico resultou em alta para enfermaria e posteriormente para o domicílio.
Gráfico 1 - Gastos do Paciente 1 por dia de internação na UTI comparados a sua média de gasto diária.

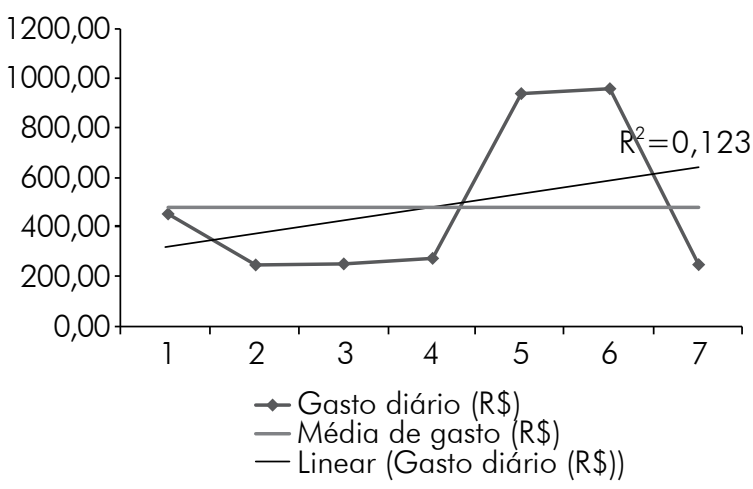

Observa-se no Gráfico 2 que nos primeiros quatro dias há um gasto superior a média diária. Isto se deu em decorrência do início do tratamento do paciente que contou com procedimentos para confirmar o diagnóstico inicial. Após o quinto dia de internação na UTI houve uma estabilidade hemodinâmica, proporcionando uma queda nos gastos diários, que consequentemente ficaram inferiores à média.

Gráfico 2 - Gastos do Paciente 2 por dia de internação na UTI comparados a sua média de gasto diária.

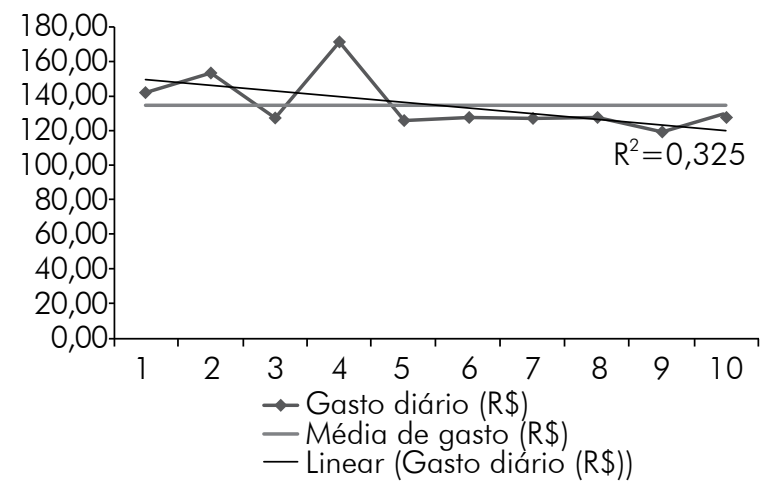

O Gráfico 3 refere-se ao paciente com 68 anos, do sexo masculino, apresentando permanência de internação na UTI de 73 dias, evoluindo a óbito.

Nos primeiros 40 dias de internação na UTI, o gasto diário permaneceu inferior à média, devido à estabilidade do quadro clínico. Após o $45^{\circ}$ dia de internação, houve um pico de elevação que coincide com a instabilidade hemodinâmica do paciente, sessóes diárias de hemodiálise, realização de procedimentos invasivos, uso de antibiótico de última geração, aminas e doses elevadas de sedação. Houve ainda diminuição brusca nos gastos, seguida de rápida elevação dos gastos que corresponde ao dia do óbito do paciente. 
Gráfico 3 - Gastos do Paciente 3 por dia de internação na UTI comparados a sua média de gasto diária.

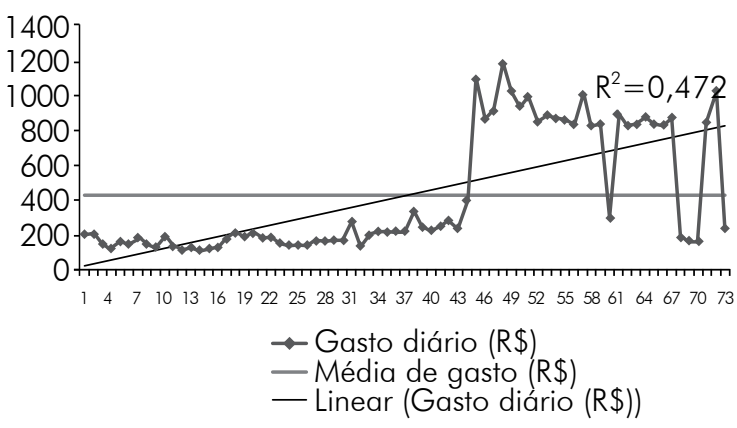

A assistência ao idoso gera gastos que põe em cheque a sustentabilidade dos planos de saúde, sendo que possivelmente apenas o Estado possa assegurar uma velhice bem assistida que efetivamente cumpra sua função [13].

Portanto, promover qualidade de vida em um ambiente de terapia intensiva torna-se questão difícil, pois num momento táo delicado, a preocupação soberana é a luta contra a morte, lançando-se mão de todos os procedimentos necessários. Nesse sentido é fundamental a participação do profissional de saúde de forma a conhecer melhor os custos referentes aos gastos no tratamento de paciente com diagnóstico de pneumonia na UTI, o que contribuirá para qualidade dos serviços prestados.

A primeira limitação deste estudo diz respeito à impossibilidade de medir as diferenças de evolução da pneumonia comunitária entre os três pacientes durante o período de internação. Por isso, as características da populaçáo estudada devem ser levadas em consideração (amostra pequena, pacientes idosos com comorbidades e outras doenças associadas) ao se tentar generalizar esses resultados para outros grupos de pacientes.

Os resultados deste estudo permitem observar que o paciente que teve maior período de internação apresentou maior média de gasto diário e este gasto esteve associado ao agravamento do quadro clínico. Outros estudos com metodologia de ensaio clínico controlado se fazem necessários.

\section{Conclusão}

Este estudo fez com que emergisse a necessidade de encontrar associação direta entre aumento do período de internação na UTI e custo do tratamento ao maior risco de mortalidade.

\section{Referências}

1. Diretrizes brasileiras para tratamento das pneumonias adquiridas no hospital e das associadas à ventilação mecânica - 2007. J Bras Pneumol 2007;33(Supl 1):S1- 30.

2. Wong LLR, Carvalho JAM. O rápido processo de envelhecimento populacional no Brasil: sérios desafios para as políticas públicas. Rev Bras Estud Pop 2006; 23:5-26.

3. Melione LPR, Mello-Jorge MHP. Gastos do Sistema Único de Saúde com internaçóes por causas externas em São José dos Campos, São Paulo, Brasil. Cad Saúde Pública 2008;24(8):1814-24.

4. Kanamura AH. Viana ALD'A. Gastos elevados em plano privado de saúde: com quem e em quê. Rev Saúde Pública 2007;41(5):814-20.

5. Vanni T, Luz PM, Ribeiro RA, Novaes HMD, Polanczyk CA. Avaliação econômica em saúde: aplicações em doenças infecciosas. Cad Saúde Pública 2009;25(12):2543-52.

6. Oliveira FA, Reis MA, Castro ECC, Cunha SFC, Teixeira VPA. Doenças infecciosas como causas de morte em idosos autopsiados. Rev Soc Bras Medicina Tropical 2004;37: 33-6.

7. Godoy DV, Dal Zotto C, Bellicanta J, Weschenfelder RF, Nacif SB. Doenças respiratórias como causa de internaçóes hospitalares de pacientes do Sistema Único de Saúde num serviço terciário de clínica médica na região nordeste do Rio Grande do Sul. J. Pneumologia 2001;27(4):193-8.

8. Drummond M, Manca A, Sculpher M. Increasing the generalizability of economic evaluations: recommendations for the design, analysis, and reporting of studies. Int J Technol Assess Health Care 2005;21:165-71.

9. Serra Negra CA, Serra Negra EM. Custo hospitalar: uma reflexão sobre implantação e necessidades. Contab Vista 2001;12(1):31-56.

10. Rodrigues VA, Perroca MG, Jericó MC. Glosas hospitalares: importância das anotaçóes de Enfermagem. Arq Ciênc Saúde 2004;11(4):210-4.

11. Francisco IMF, Castilho V. A enfermagem e o gerenciamento de custos. Rev Esc Enferm USP 2002;36(3):240-4.

12. Brasil. Ministério da Saúde. Resolução n.196, de 10 de outubro de 1996. Aprova diretrizes e normas regulamentadoras de pesquisas envolvendo seres humanos. Brasília: MS; 1996.

13. Berenstein CK, Wajnman S. Efeitos da estrutura etária nos gastos com internação no Sistema Único de Saúde: uma análise de decomposição para duas áreas metropolitanas brasileiras. Cad Saúde Pública 2008;24(10):2301-13.

14. Krauss-Silva L. Avaliação tecnológica em saúde: questôes metodológicas e operacionais. Cad Saúde Pública 2004;20Suppl2:S199-207.

15. Silva LCC. Condutas em pneumologia. Rio de Janeiro: Revinter; 2001.

16. Corrêa RA, Lopes RM, Oliveira LMG, Campos FTAF, Reis MAS, Rocha MOC. Estudo de casos hospitalizados por pneumonia comunitária no período de um ano. J Pneumol 2001;27(5):243-8.

17. Cesconetto A, Lapa LS, Calvo MCM. Avaliação da eficiência produtiva de hospitais do SUS de Santa Catarina, Brasil. Cad Saúde Pública 2008;24(10):2407-17. 